\title{
Repeated central administration of selegiline attenuated morphine physical dependence in rat
}

\author{
Alireza Parvizpour ${ }^{1}$, Mohammad Charkhpour ${ }^{1}$, Bohlool Habibi-asl ${ }^{1}$, \\ Mobin Shakhsi ${ }^{1}$, Majid Ghaderi², Kambiz Hassanzadeh ${ }^{3,4}$ \\ ${ }^{1}$ Department of Pharmacology and Toxicology, Faculty of Pharmacy, Tabriz University of Medical Sciences, \\ Tabriz, Iran \\ ${ }^{2}$ Department of Biology, Faculty of Science, Islamic Azad University, Sanandaj Branch, Sanandaj, Iran \\ ${ }^{3}$ Cellular and Molecular Research Center, Kurdistan University of Medical Sciences, Sanandaj, Iran \\ ${ }^{4}$ Department of Physiology and Pharmacology, Faculty of Medicine, Kurdistan University of Medical Sciences, \\ Pasdaran Street, 66186-34683 Sanandaj, Iran
}

Correspondence: Kambiz Hassanzadeh, e-mail: kambizhassanzadeh@gmail.com

\begin{abstract}
:
Background: Long-term exposure to opiates induces physical dependence; however, the neurobiological mechanisms of this phenomenon are not completely clear. The purpose of this study was to evaluate the effects of systemic and intracerebroventricular (icv) administration of selegiline (a selective inhibitor of monoamine oxidase B) on the morphine withdrawal syndrome in rats.

Methods: To this aim, adult male Sprague Dawley rats were selected randomly, and then growing doses of morphine were administered subcutaneously at an interval of $12 \mathrm{~h}$ for nine days with the intention of inducing dependency. Nine days after, only the morning dose of morphine was administered, followed by systemic or central injection of saline or selegiline. Later, naloxone was injected after $30 \mathrm{~min}$ and withdrawal signs recorded for a period of $60 \mathrm{~min}$.

Results: Results showed failure of systemic administration of selegiline in changing the withdrawal symptoms; nevertheless, $i \mathrm{cv}$ injection attenuated the withdrawal signs significantly.

Conclusion: In conclusion we found that central administration of selegiline attenuated morphine withdrawal symptoms
\end{abstract}

Key words:

morphine, physical dependence, selegiline, withdrawal symptoms

\section{Introduction}

Long term administration of opioids can alter the central pain-related systems and results in opioid dependence. Dependence is a behavioral state requiring con- tinued drug administration to avoid a series of aversive withdrawal syndromes. Owing to its involvement in several mental and neurological disorders, dopamine is one of the most intensely studied neurotransmitters in the brain. Dopaminergic neurons, for example, play a crucial role in the reward system that con- 
trols learning of many specific behaviors [29]. Neurochemical changes in the nucleus accumbens following systemic morphine administration in rats have been reported by Rada et al., who showed an increase in dopamine and a simultaneous decrease in acetylcholine after a single dose of the drug administration [29]. In contrast, naloxone administration in rats rendered unresponsive or tolerant by long-term effects of morphine, resulted in decreased dopamine and increased acetylcholine levels [29]. In the meantime, Sepúlveda et al. demonstrated an increased release of glutamate in the nucleus accumbens of morphine-tolerant rats following naloxone administration [33]. Drugs of abuse have been shown to produce long-term changes in the dopaminergic system, which may have important implications for the design of treatment strategies. There have been reported changes in dopamine efflux in brain slices studied 3-5 days after the onset of morphine withdrawal [5]. In brain slices obtained from the striatum, electrically evoked efflux of dopamine is diminished for up to 3 weeks following withdrawal from chronic morphine treatment [36]. In addition, our recent studies have shown that the neuroprotective agents could prevent morphine-induced tolerance and dependence $[8,10$, 12, 14-16]. Selegiline, an irreversible and selective inhibitor of monoamine oxidase B (MAO-B), has trophic and neuroprotective effects on dopaminergic neurons, which appear to be independent of monoamine oxidase inhibition [30]. The latter mode of action may explain the ability of chronic treatment with selegiline to enhance dopaminergic function in rats [20]. Because of evidence for decreased dopaminergic function during the withdrawal syndromes associated with opiates, this study aims to investigate the effects of pre-treatment with selegiline on morphineinduced withdrawal symptoms either intraperitoneal (ip) or intracerebroventricular (icv).

\section{Materials and Methods}

\section{Animals}

In our study, male Wistar rats were utilized, weighing between 250-300 g. They were kept in a controlled temperature room $\left(24 \pm 0.5^{\circ} \mathrm{C}\right)$ and maintained on a 12-h light/dark cycle while having free access to food and water. All experiments were executed in accordance with the Guide for the Care and Use of Laboratory Animals (National Institutes of Health Publication No. 85-23, revised 1985) and were approved by the Research and Ethics Committee of the Tabriz University of Medical Sciences.

\section{Experimental groups}

Rats were randomly divided into 11 groups:

1) One chronically normal saline solution $(1 \mathrm{ml} / \mathrm{kg}$ bw, ip) treated group $(\mathrm{n}=8)$.

2) One chronically subcutaneously $(s c)$ morphine and saline solution $(1 \mathrm{ml} / \mathrm{kg}$, ip $)$ treated group $(\mathrm{n}=8)$ : Control for systemic administration of selegiline.

3) One chronically morphine $(s c)$ and saline solution $(10 \mu \mathrm{l} / \mathrm{rat}, i c v)$ treated group $(\mathrm{n}=8)$ : Control for $i c v$ administration of selegiline

4) Three chronically morphine ( $s c$ ) and selegiline $(10,20$ or $40 \mathrm{mg} / \mathrm{kg} \mathrm{bw}, i p)$ treated groups $(\mathrm{n}=8)$.

$5)$ Three chronically morphine $(s c)$ and selegiline $(25,50$ or $100 \mu \mathrm{g} / 5 \mu \mathrm{l} / \mathrm{rat}, i c v)$ treated groups $(\mathrm{n}=8)$.

6) Two chronically selegiline (40 mg/kg bw, ip) or selegiline $(50 \mu \mathrm{g} / 5 \mu \mathrm{l} / \mathrm{rat}, i c v)$ alone treated groups $(\mathrm{n}=8)$.

\section{Intracerebroventricular cannula implantation}

For icv treatment, rats were anesthetized with sodium pentobarbital (50 mg/kg bw, ip) (Merck, Germany) and stereotaxically implanted with a stainless steel guide cannula (23 gauge) into the lateral cerebral ventricle (coordinates, $-0.8 \mathrm{~mm}$ posterior, $-1.3 \mathrm{~mm}$ midline to lateral and $3.5 \mathrm{~mm}$ ventral ) with respect to the bregma [27]. A stainless steel guide (30 gauge) was placed into the guide cannula as a dummy cannula to maintain the patency. After surgery, a recovery period of 7 days was allowed before the beginning of the experiment. During the recovery period, animals were habituated to the testing environment. This process included being transferred to the experimental room and handled, weighed and restrained on the test platform for $1 \mathrm{~min}$ with gentle removal and replacement of the dummy cannula (twice per day). Furthermore, testing was started after a recovery period of 7 days in all groups. 


\section{Histological verification of cannula placement}

At the end of all behavioral experiments, methylene blue solution $(5 \mu 1 / \mathrm{rat}, i c v)$ was injected into animals, and then they were euthanized with pentobarbital followed by decapitation. The brain of each animal was dissected out and cut along the coronal plane to verify the placement of the guide cannula and the distribution of the methylene blue in the ventricles after the behavioral tests. Data for only those animals that displayed a uniform distribution of methylene blue in the ventricles were considered for statistical analysis. Overall, six animals were discarded because the placement of the guide cannula was incorrect.

\section{Drug treatment}

Morphine sulfate (Temad Co., Tehran, Iran) $(10 \mathrm{mg} / \mathrm{kg}$ bw, sc) was dissolved in sterile $0.9 \%$ normal saline solution and injected subcutaneously using 1-ml insulin syringes. Following this, two mode of drug administration were considered, Either selegiline (Sigma-Aldrich, Inc.) $(10,20$ and $40 \mathrm{mg} / \mathrm{kg}$ bw) was dissolved in sterile $0.9 \%$ normal saline solution and injected ip or selegiline $(25,50,100 \mu \mathrm{g} / 5 \mu \mathrm{l} / \mathrm{rat}, i c v)$ was infused using a Hamilton syringe connected to a length of PE-10 polyethylene tubing. For the $i c v$ administration, the efficacy of injection was monitored by observing the movement of a small air bubble through the tube. The volume of infusion was $5 \mu \mathrm{l} /$ site at a rate of $5 \mu \mathrm{l} / \mathrm{min}$ for each rat. Naloxone hydrochloride (Temad Co., Tehran, Iran) (4 mg/kg bw, ip) was dissolved in sterile $0.9 \%$ normal saline solution and injected $i p$ using 1-ml insulin syringes.

\section{Induction of morphine dependence}

Animals received normal saline solution $1 \mathrm{ml} / \mathrm{kg}$ $\mathrm{bw} / 12 \mathrm{~h}$, ip or normal saline solution (ip or icv) together with morphine or selegiline (sc or $i c v$ ) plus morphine. In order to induce the dependency, additive doses of morphine were administered $s c$ for nine days as follows: day 1: $5 \mathrm{mg} / \mathrm{kg} \mathrm{bw} / 12 \mathrm{~h}$, day 2, 3: $10 \mathrm{mg} /$ $\mathrm{kg} \mathrm{bw} / 12 \mathrm{~h}$, day $4,5: 15 \mathrm{mg} / \mathrm{kg}$ bw/12 h, day 6,7 : $20 \mathrm{mg} / \mathrm{kg} \mathrm{bw} / 12 \mathrm{~h}$, day 8, 9: $25 \mathrm{mg} / \mathrm{kg} \mathrm{bw} / 12 \mathrm{~h}$. On the ninth day, only the morning dose of morphine and selegiline was injected. Selegiline was injected just before the morphine injection twice a day for nine days, needless to say that the chosen dose for morphine was selected based on previous studies [2, 22].
Tab. 1. Weighing factors (WF) of different withdrawal signs

\begin{tabular}{lc}
\hline Behavior & WF \\
\hline Jumping & 4 \\
Abdomen writhing & 5 \\
Wet-dog shakes & 5 \\
Genital grooming & 5 \\
Rearing & 20 \\
\hline
\end{tabular}

\section{Induction of morphine withdrawal and measurement of behavioral signs}

In order to induce withdrawal symptoms, naloxone (4 mg/kg bw, ip) was injected on the ninth day, one hour after the morning dose of morphine injection, needless to say that the chosen dose was selected according to previous studies $[1,12]$.

Immediately after naloxone injection, the behavioral assessment of opioid withdrawal signs including jumping, rearing, genital grooming, abdomen writhing and wet-dog shake was started. Animals were studied individually in a clear plexiglass chamber $(50 \times 25 \times 15 \mathrm{~cm})$ that was placed in other dark chamber to avoid environmental perturbations. A digital camera connected to a recording computer was placed on the inner chamber to simultaneously show the rats behaviors. The reactions of each animal were evaluated by an observer who was not aware of the nature of the treatment received by that animal. The behaviors of all animals were evaluated by the same observer. As described previously, the score of each behavior was divided by weighing factor attributed to it (Tab. 1), and the results were added and came to a total withdrawal score (TWS) for each animal [3].

\section{Data analysis}

Data were expressed as the mean \pm SEM (standard error of the mean), and analyzed by one-way analysis of variance (ANOVA) followed by Tukey's post-hoc test. Therefore, $\mathrm{p}$ values of less than 0.05 were considered as significant. 


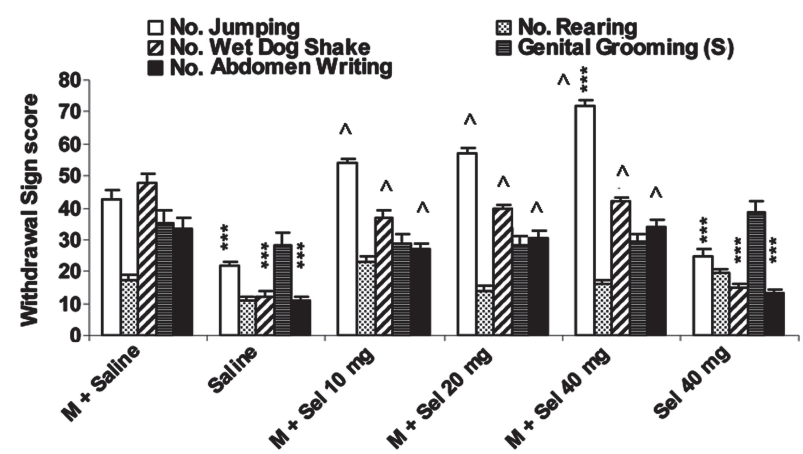

Fig. 1. Effects of repeated systemic injection of selegiline (10,20, $40 \mathrm{mg} / \mathrm{kg} \mathrm{bw}$ ) on the development of morphine dependence (withdrawal signs). Data are expressed as the mean \pm SEM. ${ }^{* * *} p<0.001$ different from control (morphine-dependent saline injected group) and $\wedge p<0.001$ different from the normal saline group. $M=$ morphine, Sel = selegiline

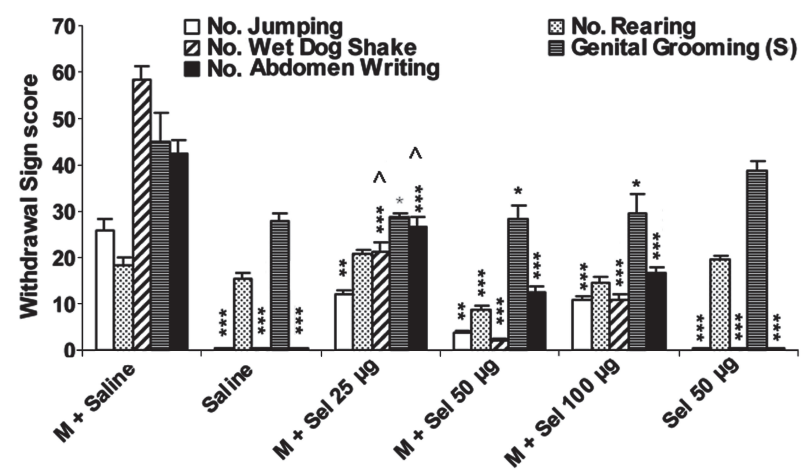

Fig. 2. Effects of repeated central injection of selegiline (10, 20 , $40 \mathrm{mg} / \mathrm{kg}$ bw) on the development of morphine dependence (withdrawal signs). Data are expressed as the mean \pm SEM. ${ }^{*} p<0.05$, ${ }^{\star \star} p<0.01,{ }^{\star \star \star} p<0.001$ different from control (morphine-dependent saline microinjected group) and $\wedge p<0.001$ different from saline group. $\mathrm{M}=$ morphine, Sel = selegiline

\section{Results}

\section{Naloxone-induced withdrawal}

Animals received repeatedly morphine twice a day for a total of nine days. Then, naloxone $(4 \mathrm{mg} / \mathrm{kg} \mathrm{bw}, i p)$ was injected in order to induce withdrawal symptoms, as shown in Figures 1 and 2. As a result, naloxone induced withdrawal signs (jumping, rearing, genital grooming, abdomen writhing and wet-dog shake) in the control group which received morphine and normal saline in comparison with normal saline. ANOVA

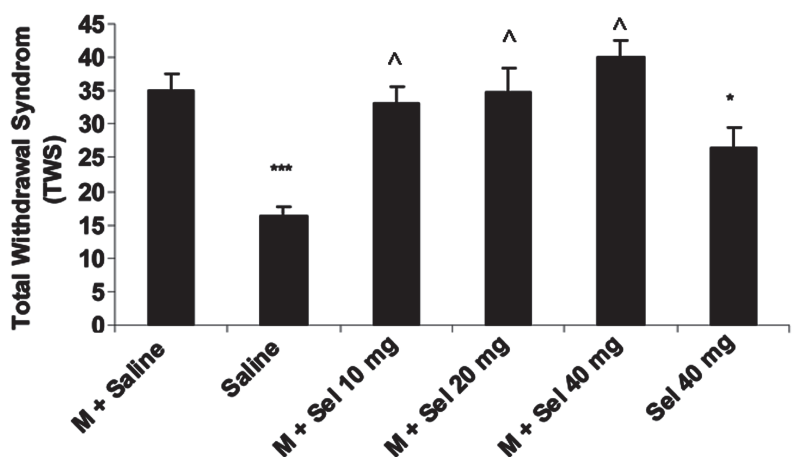

Fig. 3. Effects of repeated systemic injection of selegiline $(10,20$, $40 \mathrm{mg} / \mathrm{kg} \mathrm{bw}$ ) on the development of morphine dependence (Total Withdrawal Scores). Data are expressed as the mean \pm SEM. ${ }^{*} p<$ $0.05,{ }^{* *} p<0.001$ different from control (morphine-dependent saline injected group) and $\wedge p<0.001$ different from saline group. $M=$ morphine, Sel = selegiline

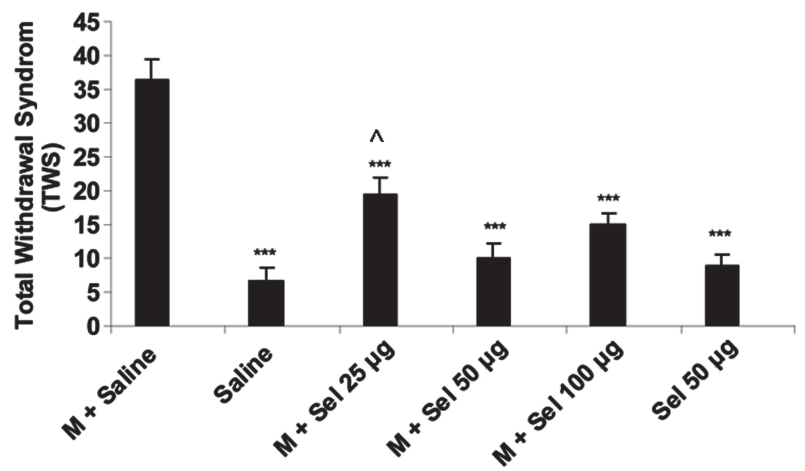

Fig. 4. Effects of repeated central injection of selegiline $(10,20$, $40 \mathrm{mg} / \mathrm{kg} \mathrm{bw}$ ) on the development of morphine dependence (Total Withdrawal Scores). Data are expressed as the mean \pm SEM. ${ }^{* \star \star} \mathrm{p}<$ 0.001 different from control (morphine-dependent saline microinjected group) and $\wedge p<0.001$ different from saline group. $M=$ morphine, Sel $=$ selegiline

test indicated that the TWS was significantly $(\mathrm{F}=$ 22.93, $\mathrm{p}<0.001$ ) greater in control group (morphine + saline) compared to saline treated animals, indicating that the animals in control group has already became tolerant (Figs. 3, 4).

\section{Effect of systemic administration of selegiline on morphine physical dependence}

The results in Figure 1 show that systemic administration of selegiline $(40 \mathrm{mg} / \mathrm{kg}$ bw, ip) increased the jumping significantly $(\mathrm{F}=3.85, \mathrm{p}<0.05)$ while it didn't change other behaviors (rearing, genital groom- 
ing, abdomen writhing and wet-dog shake). Figure 3 results show that there were no significant differences in TWS when comparing morphine injected group with morphine plus selegiline treated ones. Our findings in both Figures (1 and 3) demonstrated no significant changes between selegiline alone and saline treated animals.

\section{Effect of central administration of selegiline on morphine physical dependence}

The results showed that selegiline $i c v$ administration decreased each and every withdrawal sign considerably (Fig. 2) ( $p<0.001)$. In addition, our study showed that selegiline icv administration resulted in a decrease of TWS in comparison with the control group (Fig. 4) $(\mathrm{F}=18.8, \mathrm{p}<0.001)$, besides there was no significant difference between selegiline alone and saline treated animals.

\section{Discussion}

One of the most important findings in this study was that central but not systemic selegiline administration decreased withdrawal symptoms significantly. These findings confirmed that selegiline central administration attenuates morphine dependence. Its worth mentioning that selegiline as a selective monoamine oxidase B inhibitor, has neuroprotective properties which has been confirmed by several studies, and is used for treatment of Parkinson disease [6, 19, 31]. In addition to MAO inhibitor effects, selegiline has anti-apoptotic and pro-trophic factor properties [26-35]. Selegiline seems to have anxiolytic effects [25], which could be beneficial in morphine withdrawal attenuation signs. The results of the present study show that TWS in central administration of selegiline and not the systemic one, has a significant difference compared to the saline group indicating that central administration is more effective in decreasing the withdrawal signs.

Dopaminergic neurons are believed to be particularly prone to oxidative stress due to their high rate of oxygen metabolism, low levels of antioxidants, and high iron content. Dopamine is thought to be capable of generating toxic reactive oxygen species (ROS) via both its enzymatic and non-enzymatic catabolism [13]. Dopamine oxidation can occur either spontane- ously in the presence of transition metal ions or via an enzyme-catalyzed reaction involving MAO. Oxidation of dopamine via MAO generates a spectrum of toxic species including $\mathrm{H}_{2} \mathrm{O}_{2}$, oxygen radicals, semiquinones, and quinines [7]. Since selegiline is capable of MAO-B inhibition, it can raise dopamine levels in the human brain by preventing dopamine metabolism [39]. Selegiline is examined as a relapseprevention treatment for cocaine abuse, as well as a safe and efficient adjunctive treatment in behavioral counseling for smoking cessation [4-24]. What's more important is that it is not believed to cause dependence in animals [32].

Role of selegiline metabolism is important and should be considered, when comparing systemic and central administration of selegiline effects on withdrawal signs. From the other side, in vivo studies suggest that selegiline is metabolized mainly by the cytochrome P-450 system, resulting in identified metabolites such as desmethylselegiline, (-)-methamphetamine and (-)-amphetamine [18]. These agents can increase the activity of adrenergic pathways which has a key role in the occurrence of withdrawal symptoms and the efficacy of selegiline on physical dependence, which might be affected by metabolites action, while central injection of selegiline may indicate the specific effects of drug without metabolite interference.

Several lines of evidence suggest that N-methylD-aspartate (NMDA) receptor has a key role in morphine tolerance and dependence. Furthermore, previous studies showed that $i c v$ glutamate or NMDA administration generates withdrawal signs in morphinedependent rats [37], yet excitatory synaptic input to ventral tegmental area (VTA) mediated by glutamate is a key component of the regulation effects of dopaminergic cells. Furthermore, various animals studies suggest that co-administration of NMDA receptor antagonists prevent the development of morphine induced tolerance and dependence [1, 9, 11, 38]. Based on previous studies, selegiline but not pargyline administration (a member of MAO inhibitors) prevented NMDA receptor activation-induced cell death, suggesting that the protection provided by selegiline occurs independently of its MAO-B inhibitory effects [22].

Additionally, it has been shown that selegiline can protect dissociated PC12 cells (cell line derived from pheochromocytoma of the rat adrenal medula) from cytotoxicity of glutamate, in a process involving glu- 
tathione depletion and oxidative events [28]. Another significant recent study in the field of organotypic slice cultures reported that selegiline rescued dopaminergic neurons from NMDA $(100 \mathrm{mM})$ toxicity [34]. Moreover, selegiline has been shown to augment transcription of neurotrophic factors in cultured astrocytes including brain-derived neurotrophic (BDNF) and ciliary neurotrophic factors [21]. Finally, monoamine oxidase-B inhibition may mediate aforementioned protective effect of selegiline. In this context, Kashii et al. reported that exogenous dopamine protect cultured retinal neuronal cells against NMDA neurotoxicity [18].

In conclusion, the results of this study showed that repeated central administration of selegiline inhibits the development of morphine dependence. A single dose of $50 \mu \mathrm{g}$ proved to be the most effective one, although when different doses were administered no significant differences were observed on morphine physical dependence. All of these pharmacological actions may be relevant to the potential therapeutic effect of selegiline on morphine physical dependence. However, further investigations are required to clarify possibility of dependency in attenuation of morphine physical dependence by selegiline compared with its monoamine oxidase-B inhibitory action.

\section{References:}

1. Asl BH, Hassanzadeh K, Khezri E, Mohammadi S: Evaluation the effects of dextromethorphan and midazolam on morphine induced tolerance and dependence in mice. Pak J Biol Sci, 2008, 11, 1690-1695.

2. Charkhpour M, Nayebi AM: Evaluation of the role of 5-HT1A receptors in dorsal and median raphe nuclei on the morphine withdrawal syndrome in rat. Eur Neuropsychopharmacol, 2006, 16, S510-S511.

3. Dizgah IM, Karimian SM, Zarrindast MR, Sohanaki H Attenuation of morphine withdrawal signs by a D1 receptor agonist in the locus coeruleus of rats. Neuroreport, 2005, 16, 1683-1686.

4. George TP, O'Malley SS: Current pharmacological treatments for nicotine dependence. Trends Pharmacol Sci, 2004, 25, 42-48.

5. Ghosh S, Patel AH, Cousins M, Grasing K: Different effects of opiate withdrawal on dopamine turnover, uptake, and release in the striatum and nucleus accumbens. Neurochem Res, 1998, 23, 875-885.

6. Goverdhan P, Sravanthi A, Mamatha T: Neuroprotective effects of meloxicam and selegiline in scopolamineinduced cognitive impairment and oxidative stress. Int J Alzheimers Dis, 2012, 974013.
7. Graham DG, Tiffany SM, Bell WRJr, Gutknecht WF: Autoxidation versus covalent binding of quinones as the mechanism of toxicity of dopamine, 6-hydroxydopamine, and related compounds toward C1300 neuroblastoma cells in vitro. Mol Pharmacol, 1978, 14, 644-653.

8. Habibi-Asl B, Alimohammadi B, Charkhpour M, Hassanzadeh K: Evaluation the effects of systemic administration of minocycline and riluzole on tolerance to morphine analgesic effect in rat. Pharma Sci (Journal of Faculty of Pharmacy, Tabriz University of Medical Sciences), 2009, 15, 205-212.

9. Habibi-Asl B, Hassanzadeh K: Effects of ketamine and midazolam on morphine induced dependence and tolerance in mice. DARU, 2004, 12, 101-105.

10. Habibi-Asl B, Hassanzadeh K, Charkhpour M: Central administration of minocycline and riluzole prevents morphine-induced tolerance in rats. Anesth Analg, 2009, 109, 936-942.

11. Habibi-Asl B, Hassanzadeh K, Moosazadeh S: Effects of ketamine and magnesium on morphine induced tolerance and dependence in mice. DARU, 2005, 13, 110-115.

12. Habibi-Asl B, Hassanzadeh K, Vafai H, Mohammadi S: Development of morphine induced tolerance and withdrawal symptoms is attenuated by lamotrigine and magnesium sulfate in mice. Pak J Biol Sci, 2009, 12, 798-803.

13. Halliwell B. Reactive oxygen species and the central nervous system: J Neurochem, 1992, 59, 1609-1623.

14. Hassanzadeh K, Habibi-Asl B, Farajnia S, Roshangar L: Minocycline prevents morphine-induced apoptosis in rat cerebral cortex and lumbar spinal cord: A possible mechanism for attenuating morphine tolerance. Neurotox Res, 2011, 19, 649-659.

15. Hassanzadeh K, Habibi-asl B, Roshangar L, Nemati M, Ansarin M, Farajnia S: Intracerebroventricular administration of riluzole prevents morphine-induced apoptosis in the lumbar region of the rat spinal cord. Pharmacol Rep, 2010, 62, 664-673.

16. Hassanzadeh K, Roshangar L, Habibi-asl B, Farajnia S, Izadpanah E, Nemati M, Arasteh M, Mohammadi S: Riluzole prevents morphine-induced apoptosis in rat cerebral cortex. Pharmacol Rep, 2011, 63, 697-707.

17. Heinonen EH, Anttila MI, Lammintausta RA: Pharmacokinetic aspects of 1-deprenyl (selegiline) and its metabolites. Clin Pharmacol Ther, 1994, 56, 742-749.

18. Kashii S, Takahashi M, Mandai M, Shimizu H, Honda Y, Sasa M, Ujihara $\mathrm{H}$ et al.: Protective action of dopamine against glutamate neurotoxicity in the retina. Invest Ophthalmol Vis Sci, 1994, 35, 685-695.

19. Kitani K, Minami C, Isobe K, Maehara K, Kanai S, Ivy GO, Carrillo MC: Why (-) deprenyl prolongs survivals of experimental animals: increase of anti-oxidant enzymes in brain and other body tissues as well as mobilization of various humoral factors may lead to systemic anti-aging effects. Mech Ageing Dev, 2002, 123, 1087-1100.

20. Knoll J, Miklya I: Multiple, small dose administration of (-)deprenyl enhances catecholaminergic activity and diminishes serotoninergic activity in the brain and these effects are unrelated to MAO-B inhibition. Arch Int Pharmacodyn Ther, 1994, 328, 1-15.

21. Mizuta I, Ohta M, Ohta K, Nishimura M, Mizuta E, Hayashi K, Kuno S: Selegiline and desmethylselegiline 
stimulate NGF, BDNF, and GDNF synthesis in cultured mouse astrocytes. Biochem Biophys Res Commun, 2000, 279, 751-755.

22. Nayebi MA, Asadi M: Effect of buspirone on morphine withdrawal syndrome in mice. Pharma Sci (Journal of Faculty of Pharmacy, Tabriz University of Medical Sciences), 2009, 14, 229-235

23. Mytilineou C, Radcliffe P, Leonardi EK, Werner P, Olanow CW: L-deprenyl protects mesencephalic dopamine neurons from glutamate receptor-mediated toxicity in vitro. J Neurochem, 1997, 68, 33-39.

24. Newton TF, Kalechstein A, Beckson M, Bartzokis G, Bridge TP, Ling W: Effects of selegiline pretreatment on response to experimental cocaine administration. Psychiatry Res, 1999, 87, 101-106.

25. Nowakowska E, Kus K, Chodera A, Rybakowski J: Investigating potential anxiolytic, antidepressant and memory enhancing activity of deprenyl. J Physiol Pharmacol, 2001, 52, 863-873.

26. Paterson IA, Tatton WG: Antiapoptotic actions of monoamine oxidase B inhibitors. Adv Pharmacol, 1998, 42, 312-315.

27. Paxinos G, Watson C: The rat brain in stereotaxic coordinates, $4^{\text {th }}$ edn., Academic Press, London, 1998.

28. Pereira CM, Oliveira CR: Glutamate toxicity on a PC12 cell line involves glutathione (GSH) depletion and oxidative stress. Free Radic Biol Med, 1997, 23, 637-647.

29. Rada P, Mark GP, Pothos E, Hoebel BG: Systemic morphine simultaneously decreases extracellular acetylcholine and increases dopamine in the nucleus accumbens of freely moving rats. Neuropharmacology, 1991, 30, 1133-1136.

30. Salo PT, Tatton WG: Deprenyl reduces the death of motoneurons caused by axotomy. J Neurosci Res, 1992, 31, 394-400.

31. Schapira AH, Olanow CW: Neuroprotection in Parkinson disease: mysteries, myths, and misconceptions. JAMA, 2004, 291, 358-364.
32. Schneider LS, Tariot PN, Goldstein B: Therapy with 1-deprenyl (selegiline) and relation to abuse liability. Clin Pharmacol Ther, 1994, 56, 750-756.

33. Sepúlveda J, Astorga JG, Contreras E: Riluzole decreases the abstinence syndrome and physical dependence in morphine-dependent mice. Eur J Pharmacol, 1999, 379, 59-62.

34. Shimazu S, Katsuki H, Akaike A: Deprenyl rescues dopaminergic neurons in organotypic slice cultures of neonatal rat mesencephalon from N-methyl-D-aspartate toxicity. Eur J Pharmacol, 1999, 377, 29-34.

35. Shimazu S, Tanigawa A, Sato N, Yoneda F, Hayashi K, Knoll J: Enhancer substances: selegiline and R-(-)-1-(benzofuran-2-yl)-2-propylaminopentane [(-)-BPAP] enhance the neurotrophic factor synthesis on cultured mouse astrocytes. Life Sci, 2003, 72, 2785-2792.

36. Tjon Tien Ril HK, De Vries TJ, Wardeh G, Hogenboom F, Mulder AH, Schoffelmeer AN: Long-lasting reciprocal changes in striatal dopamine and acetylcholine release upon morphine withdrawal. Eur J Pharmacol, 1993, $235,321-322$.

37. Tokuyama S, Wakabayashi H, Ho IK: Direct evidence for a role of glutamate in the expression of the opioid withdrawal syndrome. Eur J Pharmacol, 1996, 295, 123-129.

38. Trujillo KA: The neurobiology of opiate tolerance, dependence and sensitization: mechanisms of NMDA receptor-dependent synaptic plasticity. Neurotox Res, 2002, 4, 373-391.

39. Youdim MB, Finberg JP: Pharmacological actions of 1deprenyl (selegiline) and other selective monoamine oxidase B inhibitors. Clin Pharmacol Ther, 1994, 56, 725-733.

Received: May 8, 2012; in the revised form: December 30, 2012; accepted: January 11, 2013. 